Patients with a palliative diagnosis were excluded from this study.

Results 87 patients were discharged directly home in the 6 year study period. No patients died within 30 days of discharge. The median PIM 3 score was 0.0139 (IQR 0.00720.0347). Of patients admitted diagnoses were $41 \%$ Respiratory, 33\% Peri-operative, 8\% Cardiology, 6\% Toxicology, 5\% Neurology, 5\% Gastrointestinal, 2\% Allergy and 1\% Trauma. $69(79 \%)$ of patients had a past medical history. In 34 (39\%) admissions patients had a tracheostomy. There were no discharges against medical advice. The primary team was informed of discharge for 84 (97\%) patients. A documented follow-up plan was recorded for 46 (53\%) patients. There was written communication with the patients general practitioner for $22(25 \%)$ patients. $5(6 \%)$ patients were discharged outside the hours of 9:00-17:00.

Conclusions For the most part primary teams were informed and the majority of patients had follow-up arranged, however communication with the GP was poor. Following the results of this study a standardised template was introduced at our institution to improve the discharge process.

\section{P351 THE ESTABLISHMENT OF A PAEDIATRIC METABOLIC OUTREACH CLINIC AT UNIVERSITY HOSPITAL LIMERICK}

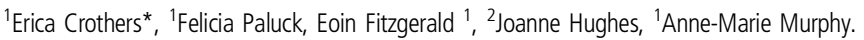
${ }^{1}$ Department of Paediatrics, University Hospital Limerick, Limerick, Ireland; ${ }^{2}$ National Centre for Inherited Metabolic Disorders, Temple Street Children's Hospital, Dublin, Ireland

\subsection{6/archdischild-2019-epa.698}

Background and aims Paediatric Medicine in Ireland is growing and changing. Care is now being delivered in alliance with the 'Hub and Spoke' model of care. Since the introduction of Newborn Screening in Ireland in 1966 there has been an increasing need for metabolic specialist care. The National Centre for Inherited Metabolic Disorders (NCIMD) is located at the Children's University Hospital, Temple Street and provides care for patients with Inherited Metabolic Disorders (IMDs). Due to the complexity of IMDs, multidisciplinary care is essential and is not only provided via Temple street, but now also through Pediatric Outreach Clinics.

The aim of this study was to describe the establishment of the Pediatric Metabolic Outreach Clinic (PMOC) at University Hospital Limerick (UHL).

Methods We describe the process involved in the establishment of the clinic, its' location and structure and the type and number of patients seen.

Results Paediatric patients with a formal diagnosis of an IMD or who were under investigation for such a diagnosis residing within the UHL catchment area were offered a review in the MOC in the Paediatric Outpatient Department at UHL in place of one of the reviews at the tertiary centre. The appointments are offered by and at the discretion of the NCIMD on a rotational basis to allow all patients living in the region the opportunity to attend and thus reduce the frequency of travel to Dublin.

The PMOC comprised a full day clinic including consultation with the Metabolic Paediatrician, Specialist Dietician and Nurse.

Since 2013 there have been 15 PMOCs at UHL.

Patients with a variety of IMDs were seen.

Conclusion With the increasing demand on Specialist Services in Ireland, outreach clinics are paramount for the future care of Paediatric patients ${ }^{4}$. Most importantly, those with IMDs in the Midwest are able to access the highest level of care closer to home.

\section{P352 A PRISM OF SELF-REFLECTION; EXPLORING THE EFFECTS OF PHYSICAL EXERCISE, REST, SOCIAL TIME, AND MINDFULNESS ON STAFF HEALTH AND WELLBEING}

${ }^{1}$ Sean Casey*, 'Jonathan Clarke, ${ }^{1}$ Alan Finan, ${ }^{2}$ Paul Gaffney, ${ }^{2}$ Aisling Hagerty. ${ }^{1}$ Paediatric Department, Cavan General Hospital, Cavan, Ireland; ${ }^{2}$ Cavan/Monaghan Primary Care Psychology Service, Cavan, Ireland

\subsection{6/archdischild-2019-epa.699}

Background There is an established body of evidence that medical professionals are at increased risk of poor mental and physical health. This negatively affects the individual practitioner, the healthcare system, and patient outcomes. To date, there have been limited interventional studies in Ireland to improve hospital staff wellbeing.

Study Design Prospective interventional study

Methods Participation was open to all staff in the paediatric department of Cavan General Hospital, a level 2 hospital in rural Ireland. A 10-week Wellness Diary was designed, in which participants recorded their weekly physical exercise, sleep hygiene, social time, and mindfulness practice.

Targets for each parameter were provided. The authors organised events and issued weekly motivational emails to help participants meet their targets. The Perceived Stress Scale (PSS) and Pittsburgh Sleep Quality Index (PSQI) were used to measure stress levels and sleep quality at baseline, Week 5, and Week 10.

Data was analyzed using Microsoft Excel 2011. Spearman coefficient was used to assess for statistically significant correlations between variables.

Results 43 participants were initially enrolled. 29 submitted diaries at Week 5, and 20 submitted at Week 10.

$55 \%$ of all participants were compliant with physical exercise targets at baseline, which remained unchanged at Week 10.

$49 \%$ of participants $(n=21)$ reported PSQI scores of $\leq 5$ (indicative of good quality sleep) at baseline, versus 59\% ( $\mathrm{n}=$ 17) by week 5 , and $89 \%(n=17)$ by week 10 . Of participants who completed the programme, $63 \%$ had PSQI scores $\leq 5$ at baseline, $68 \%$ by week 5 , and $89 \%$ by week 10 . This correlated weakly with compliance with sleep hygiene targets, though this was not statistically significant.

At baseline, participants reported having enough time for family/personal life 4.88 days of the week. This had increased to 5.05 days of the week by Week 10 .

$35 \%$ of participants $(n=15)$ used weekly mindfulness practice at baseline, versus $66 \%$ at Week 5 , and $70 \%$ at Week 10 . Of the participants who completed the programme, mean PSS scores at baseline were 15.7, 15.8 at Week 5, and 13.7 at Week 10. This correlated weakly with increased mindfulness use, though the correlation was not statistically significant.

Conclusions There are a number of limitations to our study. Only 20 participants completed all 10 weeks of the programme, limiting result generalisation. To the authors' knowledge, this is the first study of its kind in Ireland and its findings may influence future interventions. 